\title{
Assessment of Deep Vein Thrombosis (DVT) incidence among ambulatory cancer patients in Qatar: A retrospective cohort study
}

\author{
Rehab Abdelwahab ${ }^{1}$, Anas Hamad ${ }^{1}$, Reham Negm ${ }^{2}$, Nayel Al Tarawneh ${ }^{3}$ and Shereen Elazzazy ${ }^{1, *}$ \\ ${ }^{1}$ Pharmacy Department, National Center for Cancer Care \& Research, Doha, Qatar \\ 2 Oncology Hematology Department, National Center for Cancer Care \& Research, Doha, Qatar \\ ${ }^{3}$ Quality and Patient Safety Department, National Center for Cancer Care \& Research, Doha, Qatar
}

GSC Biological and Pharmaceutical Sciences, 2021, 16(03), 049-058

Publication history: Received on 16 July 2021; revised on 05 September 2021; accepted on 07 September 2021

Article DOI: https://doi.org/10.30574/gscbps.2021.16.3.0261

\begin{abstract}
Background: Thromboprophylaxis of ambulatory cancer patients is considered controversial. However, some guidelines suggest conducting VTE risk assessment using the Khorana risk assessment scoring model. This study aims to assess the incidence of Deep Vein Thrombosis (DVT) and related cancer types, focusing on the incidence of DVT in ambulatory patients actively on chemotherapy, with Khorana risk score (KRS).

Methods: The Doppler ultrasound reports over 12-month period were reviewed. A total of 205 patients were included in the study. Patients with DVT were screened for the relevant biomarkers in KRS model and any other additional risk factors. Furthermore, a comparison between ambulatory patients who developed DVT and those who did not, was carried on determining the KRS association with incidence.
\end{abstract}

Results: The incidence of DVT in ambulatory cancer patients was higher than the inpatient setting (23\% vs. 8\%). Breast cancer was the most common malignancy associated with DVT (30\%) followed by colon cancer (17\%). Chemotherapy increased the incidence of DVT in ambulatory patients (29\% vs. $13 \%$ ). Patients with KRS of $\geq 2$ were more likely to develop DVT (37.5\%).

Conclusion: This study highlights the importance of conducting a thorough DVT risk assessment for ambulatory cancer patients on chemotherapy and the need to look for KRS to reconsider additional risk factors.

Keywords: VTE; DVT; Ambulatory care; Cancer; Chemotherapy; Prophylaxis

\section{Introduction}

Venous thromboembolism (VTE) comprises of a group of conditions including deep vein thrombosis (DVT) and pulmonary embolism (PE). VTE is associated with bad mortality, morbidity rates and additional economic burden to healthcare systems [1, 2]. DVT was described through Virchow's triad due to vessels wall injury, venous stasis or hypercoagulability. Numerous risk factors are associated with the incidence of VTE such as surgery, immobilization, hereditary disorders, pregnancy and cancer [3]. The pathophysiology of VTE in cancer patients was first described in 1865 by Armand Trousseau and reinforced by different trials, all suggested that the main risk factors for DVT are hypercoagulability, vessel wall damage and vessel stasis in addition to aggressive chemotherapy regimens [1-5]. The hypercoagulable state in cancer patients is due to the high levels of tissue factor along with microparticles in the systemic circulation [5,6]. DVT might also be related to age, ethnicity, obesity, infection, renal disease, pulmonary disease or the primary site of cancer $[5,7,8]$.

\footnotetext{
* Corresponding author: Shereen Elazzazy; Email: shereen_amin@yahoo.com

Pharmacy Department, National Center for Cancer Care \& Research, Doha, Qatar
} 
The highest rates of DVT have been reported in hematological malignancies, particularly lymphoma, then lung and gastrointestinal cancers [7]. Other cancer-related factors include systemic chemotherapy, antiangiogenic agents, hormonal therapy, and some supportive therapies like Erythropoiesis-Stimulating Agents (ESA) [5,7,8]. Published data show that cancer patients represent approximately $20 \%$ of the total burden of VTE cases, while VTE is deemed to be the most common reason of death in cancer patients, as mortality rate increase 2-6 folds with the incidence of VTE [9-13].

Although VTE is considered a life-threating condition, it could still be prevented. There is a common consensus asserting the importance of VTE prophylaxis in hospitalized cancer patients [14]. Thromboprophylaxis is strongly recommended in the inpatient setting for eligible patients who had been diagnosed with cancer, unless contraindicated [4]. In the ambulatory setting, however, there are controversial debates concerning prophylaxis. Many guidelines advice against routine prophylaxis in ambulatory patients unless having additional risk factors, as compliance with VTE prophylaxis ambulatory cancer patients is more challenging and costly due to the high number of patients $[15,16]$.

Reporting of VTE events in the outpatient setting is increasing, mostly during the period of chemotherapy treatment [17]. VTE incidence reached $15 \%$ in the colorectal cancer patients on chemotherapy as reported in a retrospective cohort study [20]. In another retrospective study, VTE incidence over 12 months was $12.6 \%$ in cancer cohort group (lung, pancreas, colon/rectum, gastric, bladder, and ovary) who were commencing chemotherapy vs. $1.4 \%$ in noncancer cohort group $(\mathrm{P}<0.0001)$. Furthermore, the highest incidence of VTE was in pancreatic cancer $(19.2 \%, \mathrm{P}<0.0001)$ while the lowest was in bladder cancer (8.2\%). The majority of VTE events in the cancer group occurred soon after chemotherapy; in $18.1 \%, 47 \%$, and $72.5 \%$ within 1,3 and 6 months, respectively [21].

The most recent biomarkers for cancer-related thrombosis, which were studied mainly in ambulatory cancer patients, showed that VTE rate increased in patients with PLT $\geq 350,000 \mathrm{~mm}^{3}$ and leucocyte count $\geq 11,000 \mathrm{~mm}^{3}$. Platelet activation due to damage of vascular endothelium resulting from chemotherapy, and leukocytosis are common in cancer patients $[5,7,8,17]$.

Khorana et al developed a risk assessment model for VTE in ambulatory cancer patients who are on chemotherapy. The Khorana risk score (KRS) depends on 5 variables that are more likely related to etiology of cancer-associated thrombosis which include the site of cancer, pre-chemotherapy PLT $\geq 350,000 \mathrm{~mm}^{3}$, hemoglobin level $<10 \mathrm{~g} / \mathrm{dL}$ or the use of red-cell growth factors, pre-chemotherapy leukocyte count $\geq 11,000 \mathrm{~mm}^{3}$ and body mass index $\geq 35 \mathrm{~kg} / \mathrm{m}^{2}$. The model classifies patients into 3 categories based on their score; high risk (score $\geq 3$ ), intermediate risk (score 1-2), and low risk (score 0 ) as shown in Table 1 [17].

Table 1 Khorana predictive model for chemotherapy-associated VTE

\begin{tabular}{|l|c|}
\hline \multicolumn{1}{|c|}{ Patient characteristic } & Risk score \\
\hline Site of cancer Very high risk (stomach, pancreas) & 2 \\
\hline High risk (lung, lymphoma, gynecologic, bladder, testicular) & 1 \\
\hline Pre-chemotherapy platelet count $\geq 350,000 \mathrm{~mm}^{3} \mathrm{X} 10^{9} / \mathrm{L}$ or more & 1 \\
\hline Hemoglobin level less than $10 \mathrm{~g} / \mathrm{L}$ or use of red cell growth factors & 1 \\
\hline Prechemotherapy leukocyte count more than $11 \times 10^{9} / \mathrm{L}$ & 1 \\
\hline BMI $35 \mathrm{~kg} / \mathrm{m}^{2}$ or more & 1 \\
\hline
\end{tabular}

The PROTECHT study that was done at 62 centers in Italy and concluded that Low Molecular Weight Heparin prophylaxis halved VTE incidence in ambulatory patients with locally advanced or metastatic cancer during chemotherapy administration (2.0\% in prophylaxis group vs. $3.9 \%$ in placebo group). The prophylaxis influence was more obvious in lung cancer followed by gastrointestinal cancer patients [22].

In 2010 with the growing evidence on the importance of thromboprophylaxis for cancer patients, we in the NCCCR being the sole tertiary-center in Qatar that manages cancer realized that we need to standardize our practice in accordance with the best available evidence. Thus, a panel of experts including hematologists, oncologists, pharmacists, nurses and quality officers agreed to develop an evidence-based protocol for thromboprophylaxis. This protocol was developed and approved by our clinical practice guidelines (CPG) committee. NCCCR's VTE prophylaxis guidelines addressed the 
most important clinical issues regarding thromboprophylaxis in the inpatient and the outpatient settings. Since implementation of this protocol in the inpatient setting, the incidence of VTE during admission was significantly reduced by $66.4 \%(\mathrm{P}=0.0145)$ [23]. Yet, this was not the situation in the ambulatory setting. Therefore, in this study we are focusing on cancer patients in the ambulatory setting.

Aims and objectives: The primary objective of this study was to assess the incidence of DVT in ambulatory cancer patients at NCCCR, and to examine the association between the primary cancer types and incidence of DVT. The secondary objectives were to 1) assess the correlation between DVT in cancer patients and chemotherapy, 2) evaluate the applicability of the KRS model on our population and 3) evaluate the need to develop, based on our findings, a modified model that is more relevant to our population.

\section{Material and methods}

\subsection{Study design}

This is a retrospective case-control study. Data were extracted electronically from the radiology department records from the $1^{\text {st }}$ of January 2014 till the $31^{\text {st }}$ of December 2014. In addition, patients' profiles were reviewed via the electronic medical records (EMR) viewer and electronic clinical notes and were used as electronic sources for our data collection.

Inclusion/exclusion criteria: All ambulatory hematology and oncology cancer patients were included. While nonmalignant cases that were referred form other facilities and inpatient cases were excluded. Figure 1.
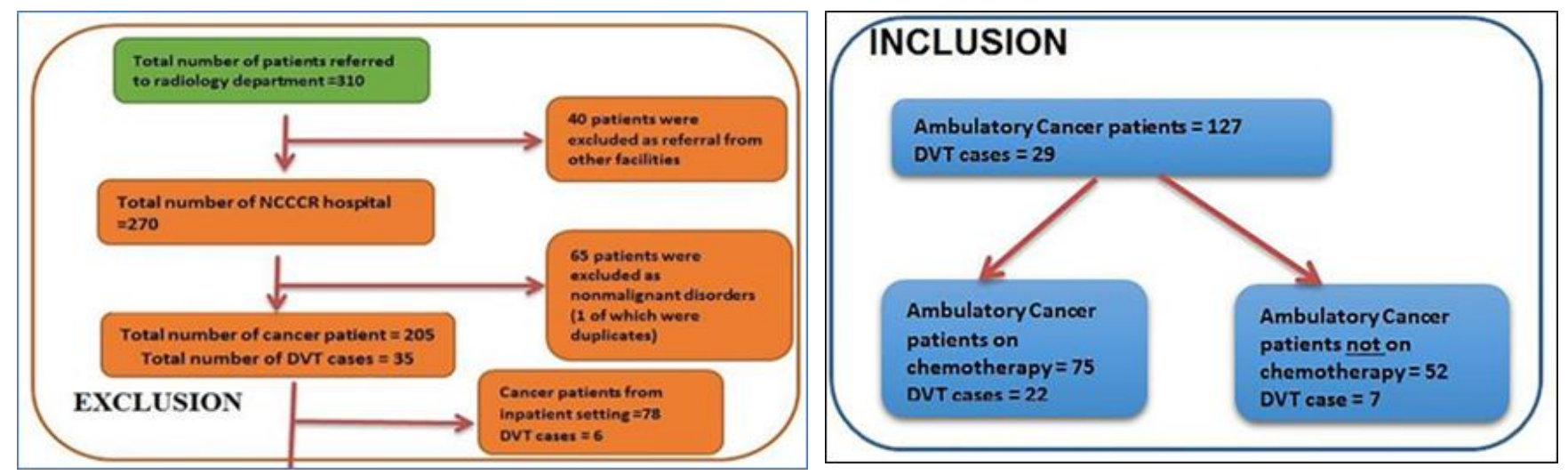

Figure 1 Flowchart diagram for patient selection (inclusion and exclusion criteria)

\subsection{Data review and analysis}

Patients` Doppler ultrasound results were screened for positive impression of DVT. Patients' medical history and the most relevant factors immediately prior to the incidence of DVT were evaluated such as gender, age, primary site of cancer, co-morbidities, history of previous VTE, last dose of chemotherapy received (if any), and concurrent administration of ESAs. Laboratory values relevant to KRS were collected including Complete Blood Count (CBC) results (platelet count, leukocyte count and hemoglobin) and BMI.

While assessing our population for the secondary outcomes, patients were stratified according to their KRS. Those with a score of 0 were considered low risk, score of 1-2 were considered intermediate risk, and score $\geq 3$ were considered high risk. Data validation was carried out by an oncology clinical pharmacy specialist and a hematologist before proceeding to the statistical analysis. Data were collected by Microsoft Excel 2010 and statistical analysis including the DVT incidence rate, odds ratio and confidence intervals was performed using SPSS version 19.

\section{Results}

A total of 310 Doppler ultrasound referrals were reviewed, 105 patients were excluded, and 205 patients were included in the study. These were distributed as 62\% (127/205) ambulatory patients vs. 38\% (78/205) inpatients. Females and elderly were more common among the ambulatory patients included $(n=127)$. More details are shown in Table 2. 
Table 2 Demographics and clinical characteristics of ambulatory cancer patients

\begin{tabular}{|l|l|l|}
\hline Parameter & $\mathbf{N = 1 2 7}$ & $\mathbf{\%}$ \\
\hline$<65$ Years & 58 & $46 \%$ \\
\hline$\geq 65$ Years & 69 & $54 \%$ \\
\hline Male & 35 & $28 \%$ \\
\hline Female & 92 & $72 \%$ \\
\hline
\end{tabular}

Data analysis showed that solid and hematology cancer cases were distributed in a ratio of 1:9 as shown (Figure 2). By comparing the inpatient vs. outpatient groups, the incidence of DVT was 8\% (6/78) vs. 23\% (29/127), respectively. Table 3 shows the DVT incidence and mortality rates, reflecting higher DVT incidence and lower mortality in the ambulatory setting.

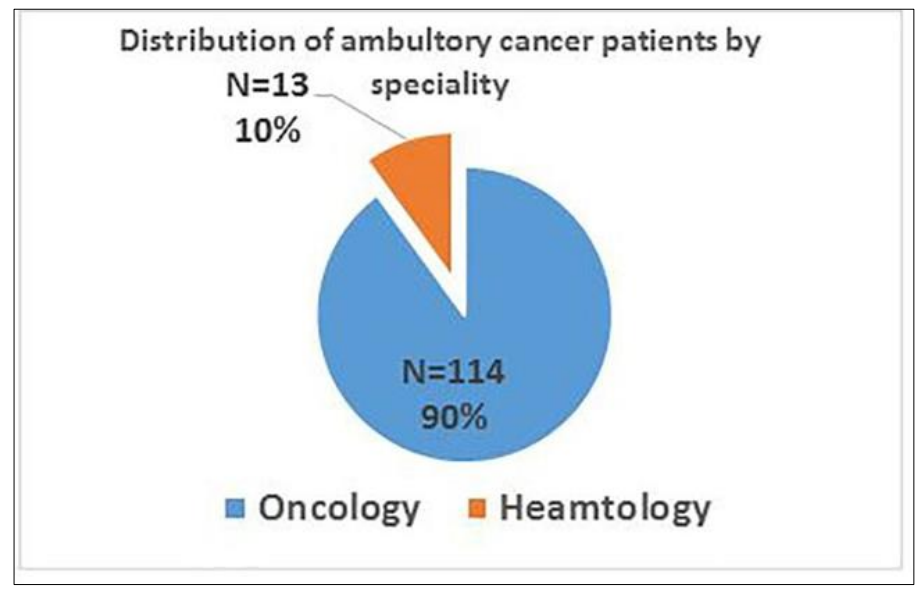

Figure 2 Distribution of malignant hematology vs. oncology cases in ambulatory cases

Table 3 Incidence of DVT and mortality rate in cancer patients (ambulatory and inpatients)

\begin{tabular}{|c|c|c|c|}
\hline $\begin{array}{c}\text { Patient distribution } \\
\mathbf{N}=\mathbf{2 0 5}\end{array}$ & $\mathbf{\%}$ & $\begin{array}{c}\text { Incidence of DVT } \\
\mathbf{N}=\mathbf{3 5}\end{array}$ & $\begin{array}{c}\text { \% of mortality rate (DVT cases) } \\
\mathbf{N}=\mathbf{1 0}\end{array}$ \\
\hline Inpatient $(\mathrm{n}=78)$ & $38 \%$ & 6 patients $(6 / 78)=8 \%$ & 2 patients $(2 / 6) 33 \%$ \\
\hline Ambulatory $(\mathrm{n}=127)$ & $62 \%$ & 29 patients $(29 / 127)=23 \%$ & 8 patients $(8 / 29) 28 \%$ \\
\hline
\end{tabular}

To focus more on the ambulatory cancer patients, the analysis included 127 patients representing ambulatory cases with solid and hematology malignancies. The overall incidence of DVT was 23\% (29/127). However, comparing the DVT incidence in patients who were actively on chemotherapy with those who were not showed that it was $29 \%$ ( $=22$ ) vs. $13 \%(\mathrm{n}=7)$, respectively [OR=2.66, CI 95\% (1.04-6.82)]. In contrast, the mortality rate was higher in the group who was not on active chemotherapy $43 \%$ (3/7) vs. $23 \%$ (5/22), as shown in table 4.

Table 4 Overview on the ambulatory cancer patients' (DVT Incidence/mortality)

\begin{tabular}{|c|c|c|c|}
\hline $\begin{array}{c}\text { Ambulatory Cancer patients } \\
(\mathbf{N}=\mathbf{1 2 7})\end{array}$ & $\mathbf{\%}$ & Incidence of DVT & \% of mortality rate \\
\hline Actively on Chemotherapy $(\mathrm{n}=75)$ & $59 \%$ & 22 patients $(22 / 75) 29 \%$ & 5 patients $(5 / 22) 23 \%$ \\
\hline Not on Chemotherapy $(\mathrm{n}=52)$ & $41 \%$ & 7 patients $(7 / 52) 13 \%$ & 3 patients (3/7) $43 \%$ \\
\hline
\end{tabular}


In ambulatory patients who developed DVT, 5-Fluorouracil and Cyclophosphamide were the most commonly used agents $(27 \%, 6 / 22)$, followed by Doxorubicin $(23 \%, 5 / 22)$, and Cisplatin and Gemcitabine $(18 \%, 4 / 22)$ for each. (Table 5)

Table 5 Most commonly used chemotherapy for ambulatory cancer patients who developed DVT

\begin{tabular}{|l|c|c|}
\hline \multicolumn{2}{|c|}{ Chemotherapy (n=22) } & No of patients $\%$ \\
\hline 5-fluorouracil & 6 & $27 \%$ \\
\hline Cyclophosphamide & 6 & $27 \%$ \\
\hline Doxorubicin & 5 & $23 \%$ \\
\hline Cisplatin & 4 & $18 \%$ \\
\hline Gemcitabine & 4 & $18 \%$ \\
\hline Docetaxel & 3 & $14 \%$ \\
\hline Irenotican & 3 & $14 \%$ \\
\hline Oxaliplatin & 2 & $9 \%$ \\
\hline Carboplatin & 2 & $9 \%$ \\
\hline Epirubicin & 2 & $9 \%$ \\
\hline Paclitaxel & 2 & $9 \%$ \\
\hline Vinorelbine & 2 & $9 \%$ \\
\hline Bevacizumab & 2 & $9 \%$ \\
\hline Cetuximab & 1 & $9 \%$ \\
\hline Vinblastine & 1 & $5 \%$ \\
\hline Vincristine & 1 & $5 \%$ \\
\hline Ifosfamide & 1 & $5 \%$ \\
\hline Bleomycin & 1 & $5 \%$ \\
\hline Dacarbazine & 1 & $5 \%$ \\
\hline Etoposide & 2 & $5 \%$ \\
\hline Methotrexate & 1 & $5 \%$ \\
\hline
\end{tabular}

Moreover, the history of previous VTE, admission within previous 60 days, KRS and DVT-related death were explored retrospectively. (Table 6)

Table 6 DVT-related Khorana risk score in ambulatory cancer patients and the related death rate

\begin{tabular}{|l|c|c|}
\hline Ambulatory cancer patients who developed DVT while on chemotherapy $(\mathbf{N}=\mathbf{2 2})$ & $\mathbf{n}$ & $\mathbf{2}$ \\
\hline Baseline laboratory value $(\mathbf{n}=\mathbf{2 2})$ & \multicolumn{2}{|l|}{} \\
\hline Khorana risk score & 9 & $41 \%$ \\
\hline 0 & 4 & $18 \%$ \\
\hline 1 & 8 & $36 \%$ \\
\hline 2 & 1 & $5 \%$ \\
\hline$>=3$ & 2 & 1 \\
\hline
\end{tabular}




\begin{tabular}{|l|c|c|}
\hline History of VTE & 6 & $27 \%$ \\
\hline Yes & 16 & $73 \%$ \\
\hline No & \multicolumn{2}{|c|}{} \\
\hline Admission within 60 day & 12 & $55 \%$ \\
\hline Yes & 10 & $45 \%$ \\
\hline No & \multicolumn{2}{|c|}{} \\
\hline DVT related death & 3 & $14 \%$ \\
\hline DVT related death & $3 \%$ \\
\hline DVT non-related death & 2 & $9 \%$ \\
\hline
\end{tabular}

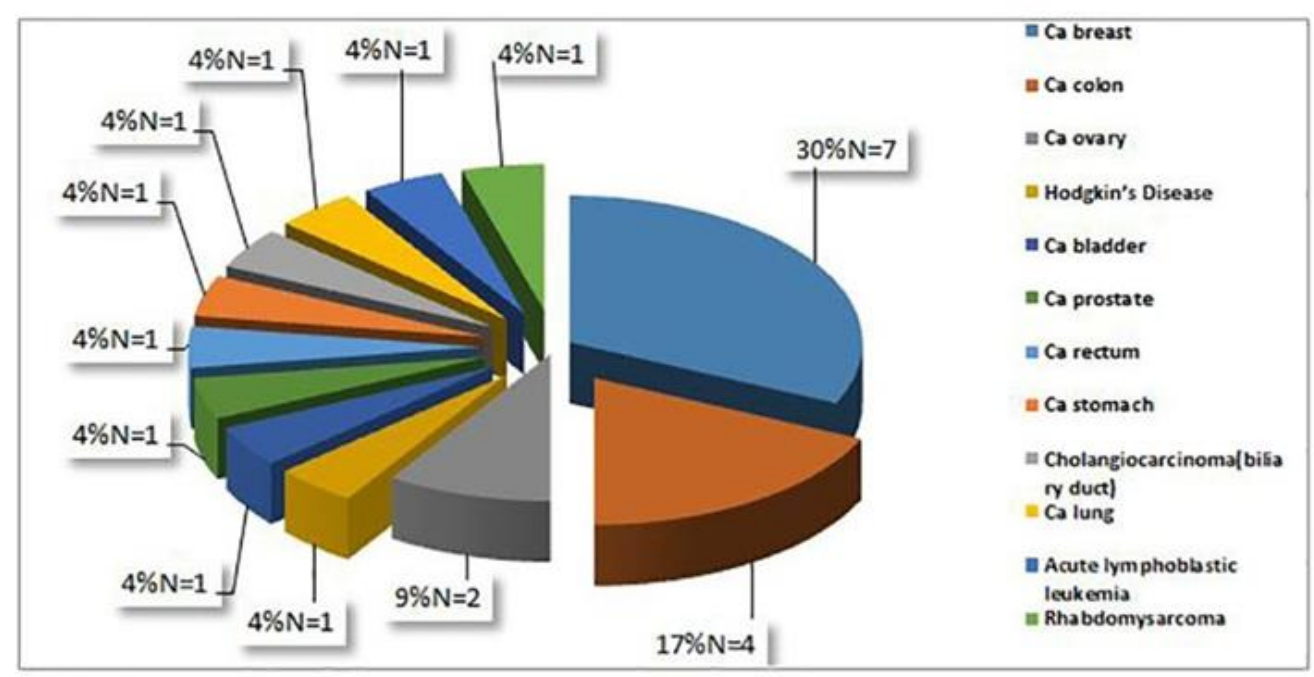

Figure 3 Distribution of ambulatory DVT patients on chemotherapy by site of tumor (n=22)

Further analysis showed that breast cancer had the highest DVT incidence (30\%) followed by colon cancer (17\%), as illustrated in figure 3. By comparing the incidence in high-risk score ( $\geq 3$ score) vs. low-intermediate risk score ( $\leq 2$ score), odds ratio (OR) was 0.79 (CI 95\% [0.07-8.07]) (Table 7, Figures 4 and 5).

The subgroup analysis showed that if we divide the DVT patients into two groups with regard to their KRS; lowintermediate risk group (i.e. score $\leq 1$ ) and high-risk group (i.e. score $\geq 2$ ), the OR for DVT incidence in the high-risk group vs. low-intermediate group would be 1.75 (CI 95\% [0.62-4.95]).

Table 7 Classification of ambulatory cancer patients on chemotherapy by risk score and DVT incidence (n=22)

\begin{tabular}{|l|c|c|c|}
\hline \multirow{2}{*}{ Risk score } & \multicolumn{2}{|c|}{ On chemotherapy } & \multirow{2}{*}{$\begin{array}{c}\text { Total } \\
\text { (N= 75) }\end{array}$} \\
\cline { 2 - 3 } & No DVT & With DVT & 26 \\
\hline 0 score (low risk) & 17 & 9 & 25 \\
\hline 1 score (intermediate risk) & 21 & 4 & 20 \\
\hline 2 score (intermediate risk) & 12 & 8 & 4 \\
\hline$\geq 3$ score *(High risk) & 3 & 1 & 75 \\
\hline Total & 53 & 22 & 2 \\
\hline
\end{tabular}




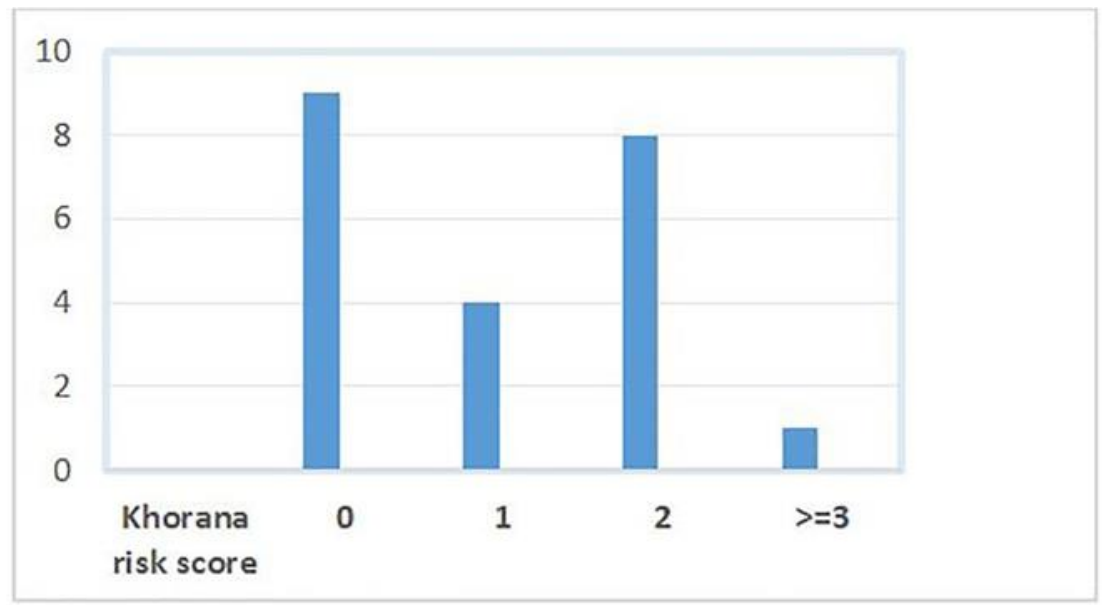

X axis represent khorona risk score for ambulatory cancer patients \& Y axis the number of patients with DVT

Figure 4 Distribution of DVT cases by Khorana risk score in ambulatory cancer patients on chemotherapy (n=22)

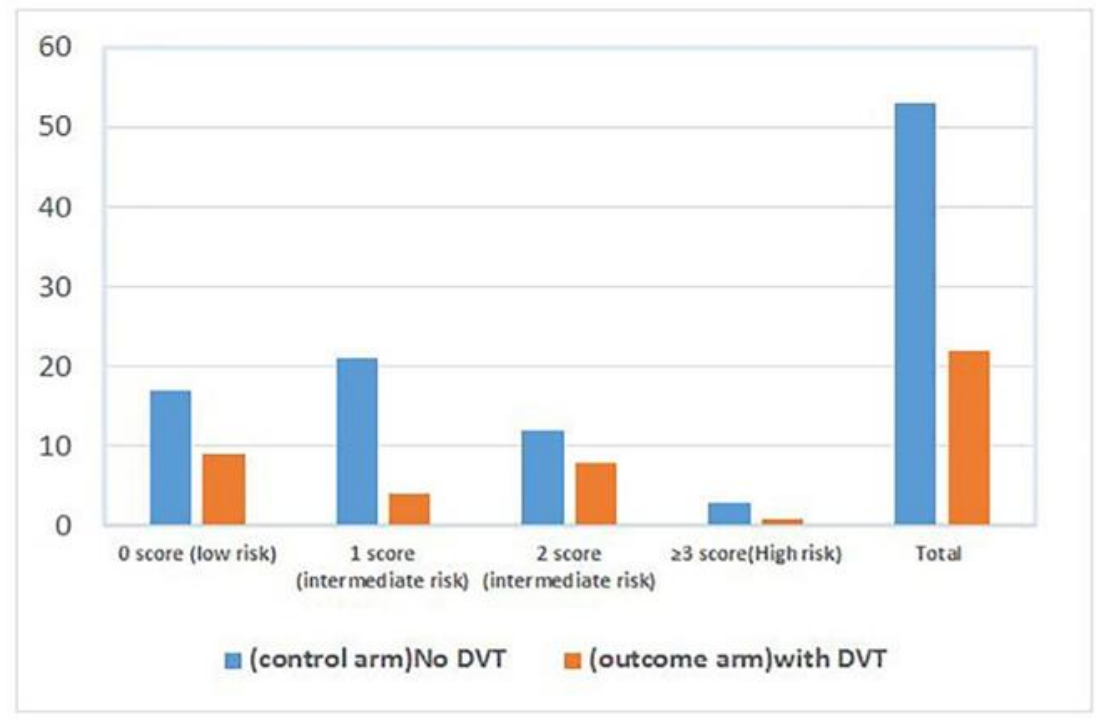

$\mathrm{X}$ axis represent khorona risk score for ambulatory cancer patients \& $\mathrm{Y}$ axis the percentage DVT cases in each risk score category

Figure 5 Distribution of ambulatory cancer patients on chemotherapy by Khorana risk score and DVT incidence

\section{Discussion}

Reducing the risk of VTE in hospitalized cancer patients was the primary clinical outcome at the time of initiating the study. However, we did not have a clear depiction regarding the existing incidence in the ambulatory setting. The results of this study are showing higher DVT incidence in the ambulatory setting (23\%) compared to the inpatient setting (8\%). Similarly, a 12-month retrospective cohort study showed that incidence of VTE in the outpatient setting (78.3\%) was higher than the inpatient setting (21.7\%), $\mathrm{P}<0.0001$ [24].

In this study, our main concern was the ambulatory cancer patients actively on chemotherapy. The DVT incidence was higher in patients who were on chemotherapy (29\%) compare to those were not (13\%). Our results are relevant to the internationally published evidence stating that the risk of VTE increases by 2-3 folds in cancer patients on chemotherapy $[25,26]$. 
Furthermore, the chemotherapy agents according to their use in the DVT cancer patients were 5-fluorouracil, cyclophosphamide, doxorubicin, cisplatin, and gemcitabine. A study that included 2,120 ambulatory patients actively receiving chemotherapy showed an increased rate of VTE amongst patients receiving Cisplatin (HR=4.20, CI 95\% [2.896.10], $\mathrm{P}<0.001)$, 5-Fluorouracil (HR=1.35, CI 95\% [0.63-2.90], $\mathrm{P}=0.004)$ and Irinotecan (HR=4.19, CI 95\% [2.90-6.04], $\mathrm{P}<0.001)$. It was clear that chemotherapy was meaningfully associated with VTE [27]. In our study, the mortality rate in DVT cases on chemotherapy was 23\% (5/22). By reviewing the reason of death, the DVT-related death rate was $14 \%$, which is higher than the rate $(9.2 \%)$ in a prospective observational study of cancer patients commencing chemotherapy regimen [28].

Our analysis showed that incidence of DVT in breast cancer was the highest representing 30\%, followed by colon cancer $17 \%$. This reflects the high prevalence of these cancer types in the general population. Our results are almost mirroring the findings of the VERITY trial, where the most common cancer types were breast, prostate, colorectal and lung with VTE incidence of 56.1\%, with the highest incidence observed for breast cancer patients (17\%) [29]. Our findings show that after colorectal cancer, bladder, ovarian and pancreatic cancer came next with DVT incidence of $5.71 \%$ each. Although those cancer types were associated with high DVT incidence similar to breast cancer, the limited number of patients in our study was the barrier to get more reliable figures. The secondary objective of this study was to find an effective strategy to categorize the ambulatory cancer patients based on their VTE risk. In 2008, KRS was developed considering that patients with score $\geq 3$ had higher risk to develop VTE [17]. KRS was further validated in other studies [30]. In our study, we calculated KRS for the ambulatory patients on chemotherapy. All subjects in both arms were assessed whether they had one or more risk factors and grouped into one of 4 categories; score 0 , score 1 , score 2 , and score $\geq 3$. We found that DVT incidence in the high-risk score group was less than the low-intermediate risk score group $(\mathrm{OR}=0.79)$. It was noted among our population that the number of DVT cases in the score 2 group was higher than score 3 group. Therefore, the high-risk group in our study was actually $\geq 2$, (OR=1.75, CI 95\% [0.62-4.95]). This will urge the need to consider the patients with score $\geq 2$ as high-risk patients, which could make them good candidates for thromboprophylaxis. We could not rationalize the DVT incidence for 13 cases in the low-intermediate risk group in a way that we could consider thromboprophylaxis or not. KRS did not give a guidance for risk assessment in that group, which warrants more focused research efforts.

\section{Conclusion}

This study highlights the importance for a thorough VTE risk assessment for ambulatory cancer patients who will be started on chemotherapy. Risk scoring model should be revised to include patients with score $\geq 2$ as a high-risk group. Breast and colon cancer patients need more attention. Clinicians are urged to discuss the risks and benefits of this intervention with their patients before deciding on thromboprophylaxis. More robust evidence is needed from prospective studies, to help in the implementation of an advanced approach for thromboprophylaxis in our patients.

\section{Compliance with ethical standards}

\section{Acknowledgments}

We would like to acknowledge HMC MRC for funding this study.

\section{Funding}

This research was funded by Hamad Medical Corporation (HMC) Medical Research Center (MRC).

\section{Disclosure of conflict of interest}

All the authors declare no conflict of interest.

\section{Author Contributions}

"Conceptualization, RA and SE; methodology, RA; software, RA; validation, RN and SE; formal analysis, RA; investigation, RA, SE and RN; resources, NT; data curation, NT and RA; writing—original draft preparation, RA; writing-review and editing, SE and AH; visualization, AH; supervision, SE; project administration, AH; funding acquisition, RA and SE. All authors have read and agreed to the published version of the manuscript.

\section{Data Availability Statement}

The data presented in this study are available on request from the corresponding author. 


\section{Abstract previous presentations}

- $\quad$ NCCCN 21-23 March 2017, published in JNCCN

- $\quad$ MASSC 22-24 June 2017, published in Supportive Care in Cancer

- AC FORUM 20-22 April 2017.

\section{Statement of ethical approval}

This study protocol was approved by the departments' heads, Hospital Research Committee (HRC), Medical Research Center (MRC) and Institutional Review Board (IRB). Ref \#MRC06868/1016.

\section{Statement of informed consent}

Patient consent was waived as this is a file review retrospective study.

\section{References}

[1] R Ruth McCaffrey, Cindy Blum. Venothrombotic Events: Evidence-based Risk Assessment, Prophylaxis, Diagnosis, and Treatment. Journal for Nurse Practitioners. 2009; 5(5): 325-333.

[2] Alisa S Wolberg, Maria M Aleman, Karin Leiderman, Kellie R Machlus. Procoagulant Activity in Hemostasis and Thrombosis: Virchow's Triad Revisited. Anesth Analg. 2012; 114(2): 275-285.

[3] Andra H James. Venous Thromboembolism: Mechanisms, Treatment, and Public. Arterioscler Thromb Vasc Biol. 2009; 29(3): 398-310.

[4] Micheal B Streiff, Bjorn Holmstorm, Dana Angelini, et al. Cancer Associated Venous Thromboembolic Disease, NCCCN Guidelines. 2015.

[5] Tarek Sousou, Alok A Khorana, James P Venous. Thromboembolism: Mechanisms, Treatment, and Public Awareness New Insights Into Cancer-Associated Thrombosis. Arteriosclerosis, Thrombosis, and Vascular Biology. 2009; 29: 316-320.

[6] E Donnellan, B Kevane BR Healey, F Ni Ainle. Cancer and venous thromboembolic disease: from molecular mechanisms to clinical management. Curr Oncol. 2014; 21(3): 134-143.

[7] Jeanet W Blom, Carine JM Doggen, Susanne Osanto, Frits R Rosendaal. Malignancies, prothrombotic mutations, and the risk of venous thrombosis. Journal of American Medical Association. 2005; 293(6): 715-722.

[8] AA Khorana, James P. Cancer and Thrombosis: Implications of Published Guidelines for Clinical Practice. Annals of Oncology. 2009; 20(10): 1619-1630.

[9] Heit JA, O'Fallon WM, Petterson TM, Lohse CM, et al. Relative impact of risk factors for deep vein thrombosis and pulmonary embolism: a population-based study. JAMA Internal Medicine. 2002; 162(11): 1245-1248.

[10] Khorana AA, Francis CW, Culakova E, Kuderer NM, et al. Thromboembolism is a leading cause of death in cancer patients receiving outpatient chemotherapy. Journal of Thrombosis and Haemostasis. 2007; 5(3): 632-634.

[11] Agnelli G, Bolis G, Capussotti L, Scarpa RM, et al. A clinical outcome-based prospective study on venous thromboembolism after cancer surgery: the @RISTOS project. Annals of Surgery. 2006; 243(1): 89-95.

[12] Helen K Chew, Theodore Wun, Danielle Harvey, Hong Zhou, et al. Incidence of venous thromboembolism and its effect on survival among patients with common cancers. JAMA Internal Medicine. 2006; 166(4): 458-464.

[13] Sørensen HT, Mellemkjaer L, Olsen JH, Baron JA. Prognosis of cancers associated with venous thromboembolism. The New England Journal of Medicine. 2000; 343(25): 1846-1850.

[14] Khorana AA, Streiff MB, Farge D, Mandala M, et al. Venous Thromboembolism Prophylaxis and Treatment in Cancer: A Consensus Statement of Major Guidelines Panels and Call to Action. Journal of Clinical Oncology. 2009; 27(29): 4919-4926.

[15] Clive Kearon, Anthony J. Comerota, Paolo Prandoni, et al. Antithrombotic Therapy and Prevention of Thrombosis,9th ed: American College of Chest Physicians Evidence-Based Clinical Practice Guidelines. 2012.

[16] Gary H Lyman, Kari Bohlke, Alok A Khorana, Nicole M Kuderer, et al. Venous Thromboembolism Prophylaxis and Treatment in Patients With Cancer: American Society of Clinical Oncology Clinical Practice Guideline Update 2014, version 2015 . 
[17] Alok A Khorana, Nicole M Kuderer, Eva Culakova et al. Development and validation of a predictive model for chemotherapy-associated thrombosis. Blood Journal. 2008; 111(10): 4902-4907.

[18] Khorana AA, Francis CW, Culakova E, Lyman GH. Risk factors for chemotherapy-associated venous thromboembolism in a prospective observational study, Cancer. 2005; 104(12): 2822-2829.

[19] Haddad TC, Greeno EW. Chemotherapy-induced thrombosis. Thrombosis Research. 2006; 118(5): 555-568.

[20] Otten HM, Mathijssen J, ten Cate H, Soesan M, et al. Symptomatic venous thromboembolism in cancer patients treated with chemotherapy: an underestimated phenomenon. JAMA Internal Medicine. 2004; 164(2): 190-194.

[21] Khorana AA, Dalal M, Lin J, Connolly GC. Incidence and predictors of venous thromboembolism (VTE) among ambulatory high-risk cancer patients undergoing chemotherapy in the United States. Cancer. 2012; 119(3): 648655.

[22] Agnelli G, Gussoni G, Bianchini C, Verso M, et al. Nadroparin for the prevention of thromboembolic events in ambulatory patients with metastatic or locally advanced solid cancer receiving chemotherapy: a randomized, placebo-controlled, double-blind study. Lancet Oncology. 2009; 10(10): 943-949.

[23] S Elazzazy, R Abd El Wahab, R Negm, MA/Wahid, et al. Clinical Outcomes of Implementing Evidence-Based Practice on Venous Thromboembolism Prevention for Cancer Patients in Qatar, A Retrospective Study. Journal of Clinical Therapeutics. 2015; 37(8): 22-23.

[24] Alok A Khorana, Mehul Dalal, Krishna Tangirala, Raymond Miao. Higher Incidence of Venous Thromboembolism in the Outpatient Versus the Inpatient Setting Among U.S. Cancer Patients. Blood Journal. 2011; 118(21): 674.

[25] Blom JW, Vanderschoot JP, Oostindiër MJ, Osanto S, et al. Incidence of venous thrombosis in a large cohort of 66 329 cancer patients: results of a record linkage study. J Thromb Haemost. 2006; 4(3): 529-535.

[26] Michael B Streiff. Association Between Cancer Types, Cancer Treatments, and Venous Thromboembolism in Medical Oncology Patients. Clinical Advances in Hematology \& Oncology. 2013; 11(6): 349-357.

[27] MA Shah, M Capanu, G Soff, T Asmis, et al. Risk factors for developing a new venous thromboembolism in ambulatory patients with non-hematologic malignancies and impact on survival for gastroesophageal malignancies. Journal of Thrombosis and Haemostasis. 2010; 8(8): 1702-1709.

[28] Khorana AA, Francis CW, Culakova E, Kuderer NM, et al. Thromboembolism is a leading cause of death in cancer patients receiving outpatient chemotherapy. Journal of Thrombosis and Haemostasis. 2007; 5(3): 632-634.

[29] Paneesha S, McManus A, Arya R, Scriven N, et al. Frequency, demographics and risk (according to tumour type or site) of cancer-associated thrombosis among patients seen at outpatient DVT clinics. Thrombosis and Haemostasis. 2009; 103(2): 338-343.

[30] Lustig DB, Rodriguez R, Wells PS. Implementation and validation of a risk stratification method at The Ottawa Hospital to guide thromboprophylaxis in ambulatory cancer Patients at intermediate-high risk for venous thrombosis. Thrombosis Research. 2015; 36(6): 1099-1102.

[31] Khorana AA, Francis CW, Culakova E, Kuderer NM, et al. Frequency, risk factors, and trends for venous thromboembolism among hospitalized cancer patients. Cancer. 2007; 110(10): 2339-2346. 